\title{
Comprehensive and Comparative Study of Different Image Fusion Techniques
}

\author{
M. Ramya Princess ${ }^{1}$, V. Suresh Kumar $^{2}$, M. Ramjan Begum ${ }^{3}$ \\ PG Student [C\&I], Dept. of EIE, Valliammai Engineering College, Chennai, Tamil Nadu, India ${ }^{1}$ \\ Assistant professor, Dept. of EIE, Valliammai Engineering College, Chennai, Tamil Nadu, India ${ }^{2,3}$
}

\begin{abstract}
Image Fusion is the process in which two or more images are combined into single composite image which can retain all important features of all original images. Image Fusion is one of the major research fields in image processing. This technique can be applied in variety of domains: Navigation, Remote Sensing, Object detection, Object recognition, etc. And it plays a major role in medical applications in order to improve diagnosis and for proper treatment planning. The main objective of image fusion is to reduce redundancy and to extract particular information. The purpose of this paper is to elaborate wide range of algorithms and their comparative study together. There are many techniques proposed by different authors in order to fuse the images and produce the clear visual of the image. And this paper also provides survey about some of the various existing techniques applied for image fusion and comparative study of all the techniques concludes the better approach for its future research.
\end{abstract}

Keywords: Wavelet Transform, DCWT, Curvelet Transform, Principal Component Analysis, Mean Square Error, Peak Signal to Noise Ratio.

\section{INTRODUCTION}

Image fusion has become a common term used within medical diagnostics and treatment. The term is used when multiple patient images are registered and overlaid or merged to provide additional information. Fused images may be created from multiple images from the same imaging modality or by combining information from multiple modalities. To get full information regarding the body organs and tissues of human, there may be by invasive or non-invasive methods, in seconds there are different modalities of medical imaging techniques, and have their respective application ranges. For instance, MRI image provides details on soft tissue with more distortion and thus bones spacing cannot be seen, while CT image provides details on denser tissue with less distortion and thus bones spacing can be seen clearly. Combining these two images will provide much more detailed information. Use of the Simple primitive technique will not recover good fused image in terms of performance parameter like peak signal to noise ratio (PSNR), Normalized correlation (NC), and Men square error (MSE). Recently, Discrete Wavelet Transform (DWT) and Principal Component Analysis (PCA), Morphological processing and Combination of DWT with PCA and Morphological techniques have been popular fusion of image. These methods are shown to perform much better than simple averaging, maximum, minimum.

Image fusion algorithms can be categorized into different levels: low, middle, and high; or pixel, feature, and decision levels. The pixel-level method works either in the spatial domain or in the transform domain. Pixel level fusion works directly on the pixels obtained at imaging sensor outputs while feature level fusion algorithms operate on features extracted from the source images. The feature-level algorithms typically segment the image into contiguous regions and fuse the regions together using their properties. The features used may be calculated separately from each image or they may be obtained by the simultaneous processing of all the images. Decision level fusion uses the outputs of initial object detection and classification as inputs to the fusion algorithm to perform the data integration. Both feature level and decision level image fusion may result in inaccurate and incomplete transfer of information. Recently researchers have recognized that it is more meaningful to combine objects or regions rather than pixels. The region based algorithm has many advantages over pixel based algorithm like it is less sensitive to noise, better contrast, less affected by miss-registration but at the cost of complexity. In order to study the differences between various existing 


\title{
International Journal of Advanced Research in Electrical, Electronics and Instrumentation Engineering
}

\author{
(An ISO 3297: 2007 Certified Organization)
}

\section{Vol. 3, Issue 9, September 2014}

techniques comprehensive and comparative study is required. This report is organized as follows: Section II presents brief description of image Fusion techniques, Section III gives Performance Measures parameter of Fusion techniques , Section IV presents performance comparison of those techniques and finally, conclusion is presented in Section V.

\section{IMAGE FUSION TECHNIQUES}

Image fusion techniques can enhance a digital image without spoiling it. The enhancement methods are of two types; namely spatial domain methods and frequency domain methods. In spatial domain techniques, we directly deal with the image pixels. The pixel values are manipulated to achieve desired enhancement. The fusion methods, such as averaging, the Brovey method, principle component analysis (PCA), and IHS based methods fall under the spatial domain approaches. In frequency domain methods, the image is first transferred in to frequency domain. It means that, the Fourier Transform of the image is computed first. All the enhancement operations are performed on the Fourier transform of the image and then the Inverse Fourier transform is performed to get the resultant image. These enhancement operations are performed in order to modify the image brightness, contrast or the distribution of the grey levels. As a consequence the pixel value (intensities) of the output image will be modified according to the transformation function applied on the input values. Pyramid Fusion Algorithm is a fusion method in the transform domain. Another important spatial domain fusion method is the high pass filtering based technique. The disadvantage of spatial domain approaches is that they produce spatial distortion in the fused image. Spectral distortion becomes a negative factor while we go for further processing such as classification problem. Spatial distortion can be very well handled by frequency domain approaches on image fusion.

\section{A. SIMPLE AVERAGE}

This algorithm is a simple way of obtaining an output image with all regions in focus. The value of the pixel $P(i, j)$ of each image is taken and added. This sum is then divided by 2 to obtain the average. The average value is assigned to the corresponding pixel of the output image. This is repeated for all pixel values.

$$
K(i, j)=\{X(i, j)+Y(i, j)\} / 2
$$

Where $X(i, j)$ and $Y(i, j)$ are two input images.

\section{B. SELECT MAXIMUM}

This algorithm chooses the in-focus regions from each input image by choosing the greatest value for each pixel, resulting in highly focused output. The value of the pixel $\mathrm{P}(\mathrm{i}, \mathrm{j}$ ) of each image is taken and compared to each other. The greatest pixel value is assigned to the corresponding pixel [2].

\section{PRINCIPAL COMPONENT ANALYSIS}

PCA is a mathematical tool which transforms a number of correlated variables into a number of uncorrelated variables [4]. The first principal component accounts for as much of the variance in the data as possible and each succeeding component accounts for as much of the remaining variance as possible. First principal component is taken to be along the direction with the maximum variance. The second principal component is constrained to lie in the subspace perpendicular of the first. Within this Subspace, this component points the direction of maximum variance. The third principal component is taken in the maximum variance direction in the subspace perpendicular to the first two and so on. The information flow diagram of PCA-based image fusion algorithm is shown in Fig.1. where $\mathrm{I}_{1}(\mathrm{x}, \mathrm{y})$ and $\mathrm{I}_{2}(\mathrm{x}, \mathrm{y})$ are the two input images which are to be fused. The following steps describe the use of PCA algorithm for fusion. 


\section{International Journal of Advanced Research in Electrical, Electronics and Instrumentation Engineering}

(An ISO 3297: 2007 Certified Organization)

Vol. 3, Issue 9, September 2014

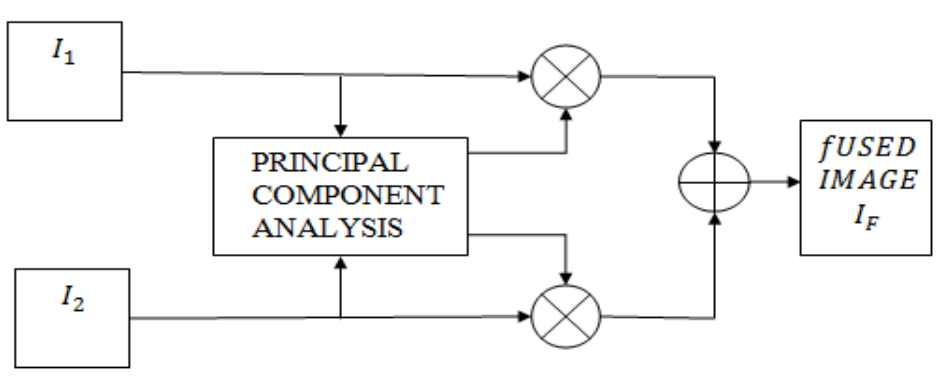

Figure 1.Information flow diagram in image fusion scheme employing PCA

1. First of all produce the column vectors, respectively, from the input image matrices.

2. Now, calculate the covariance matrix of the two column vectors formed in step 1

3. The diagonal elements of the $2 \times 2$ covariance vector would contain the variance of each column vector with itself, respectively.

4. Calculate the Eigen values and the Eigen vectors of the covariance matrix

5. Normalize the column vector corresponding to the larger Eigen value by dividing each element with mean of the Eigen vector.

6. The values of the normalized Eigen vector act as the weight values which are respectively multiplied with each pixel of the input images.

7. Sum of the two scaled matrices calculated in 6 will be the fused image $\left(\mathrm{I}_{\mathrm{F}}\right)$ matrix.

\section{DUAL TREE COMPLEX WAVELET}

The SWT only provides details in three directions for each scale. To overcome this problem, the DTCWT is proposed, which is approximately shift-invariant, directionally selective, and computationally efficient. Dual-tree of wavelet filters is used to obtain the real and imaginary parts of complex wavelet coefficients. A simple delay of one sample between the filters of the first level in each tree is conducted, and then odd-length and even-length linear-phase filters are used alternately. The filters in the two trees are just time-reverse of each other [7]. But this method fails to detect edges, curves as well as corner points.

\section{E. CURVELET}

The DWT, SWT, and DTCWT cannot capture curves and edges of images well. More reasonable bases should contain geometrical structure information when they are used to represent images. Two examples of the CVT bases are shown in Fig. 2a. Fig. 2b presents two examples of wavelet bases. From Fig. 2, it can be seen that the CVT is more suitable for the analysis of image edges, such as curve and line characteristics, than wavelet. The CVT is referred to as the "true" 2D transform. The discrete version implemented in this research uses a "wrapping" transform.

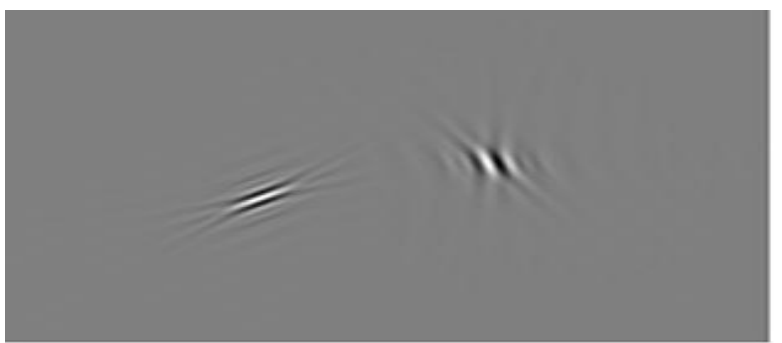

a

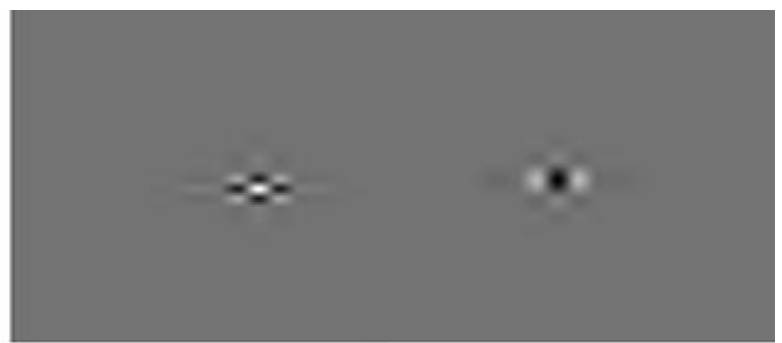

b

Figure 2.Comparison between Curvelet bases and wavelet bases. (a) Two bases of Curvelet; (b) two bases of wavelet. 


\title{
International Journal of Advanced Research in Electrical, Electronics and Instrumentation Engineering
}

\author{
(An ISO 3297: 2007 Certified Organization)
}

\section{Vol. 3, Issue 9, September 2014}

\section{F. CROSS SCALE FUSION RULE}

An optimal set of coefficients from the multiscale representations of the source images is determined by effective exploitation of neighborhood information. And this rule blends the pixel values in the monochrome source images to combine information while preserving or enhancing contrast. The general procedure of MSD-based fusion is illustrated in Fig.3. First, the source images are transformed to multiscale representations using MSD. An MSR is a pyramidal structure with successively reduced spatial resolution; it usually contains one approximation level storing low-pass coefficients and several detail levels (DETs) storing high-pass or band pass coefficients. Then, a certain fusion rule is applied to merge coefficients at different scales. Finally, an inverse MSD is applied to the fused MSR to generate the final image.

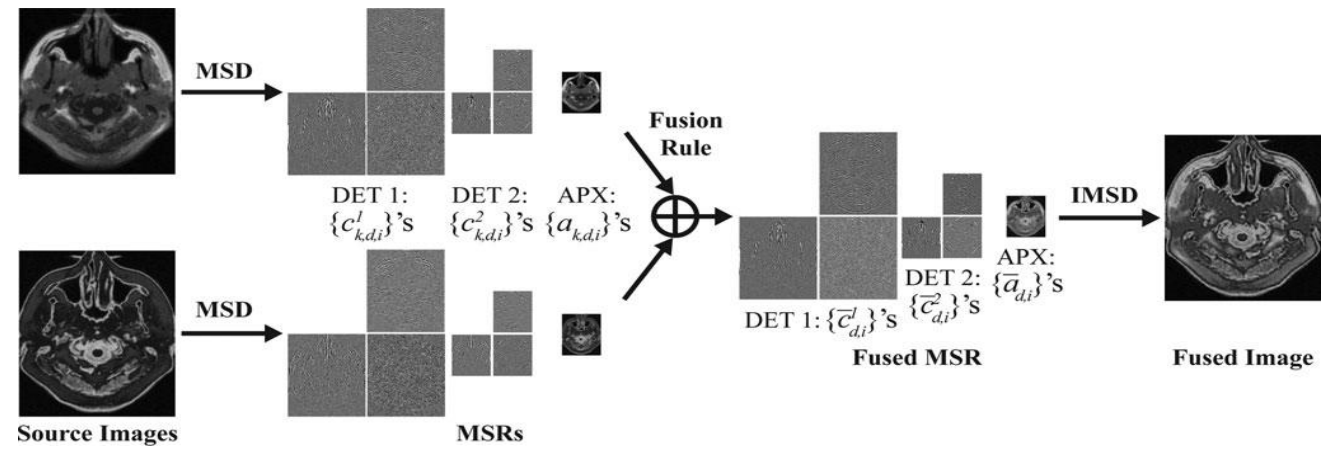

Figure 3.General procedure of MSD-based image fusion in a 2-D case

The CS fusion rule aims to pass information within and between each decomposition level to achieve intrascale and interscale consistencies so that the fused image preserves the most details from the source images while exhibiting minimal artifacts. The basic steps are: 1) pass salient information from a lower level to a higher level in an MSR until APX is reached; 2) calculate the memberships of each fused coefficient at APX using the passed salient information; 3) use these memberships to guide the coefficient selection at DETs. This method also leads to wrong diagnosis because of the wrong selection of cross scale coefficient in some cases.

\section{G. GENITIC ALGORITHM}

Image fusion using Genetic algorithm is proposed in the reference [3] for the detection of brain tumor. Preprocessing is done on the input images to reduce or suppress noise and other small fluctuations in the image. Image Enhancement is also done in preprocessing to use to sharpen image features and in turn improves the quality of the input images. After the preprocessing operations, the input images are subjected to feature extraction. Feature Extraction is used to transform the image into a set of feature. Genetic algorithm is applied in extracted features of the image to fuse the images. The block diagram of this proposed method is shown in Fig. 4. The extracted features are considered as the population of chromosomes. The fitness function is calculated for all the chromosomes. Single; two point; uniform crossovers are used to generate offsprings. The offsprings are then mutated to generate the fused image but the resultant image produced is of reduced contrast. 


\section{International Journal of Advanced Research in Electrical, Electronics and Instrumentation Engineering}

(An ISO 3297: 2007 Certified Organization)

Vol. 3, Issue 9, September 2014

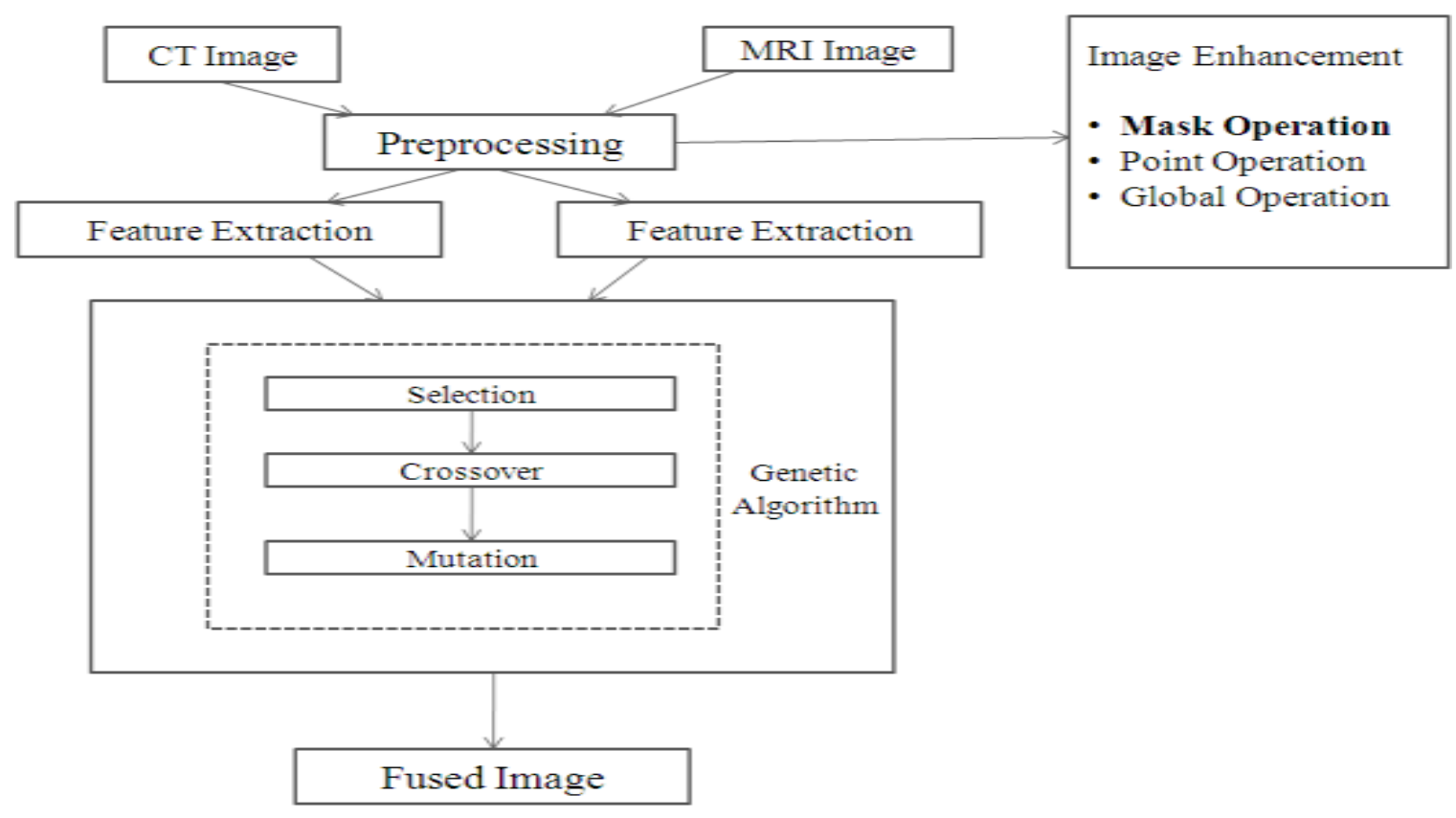

Figure 4.Image fusion using Genetic Algorithm

\section{H. PROPOSED METHOD}

In order to overcome all the limitations which are mentioned above, I would like to propose one method called Featured based information fusion using Gabor wavelet transform. The Gabor filter finds its application in feature extraction, face recognition, object detection etc. The given input image is subjected to scale and orientation so that all the features are concentrated for the detailed analysis. Now am going to choose scale as 3 and orientation as 6 , so totally I will be getting 18 Gabor features which are subjected into Independent Component Analysis for concentrating on particular tissue. By principal component analysis, the whitening matrix plays a major role. By this technique, the redundant data can be reduced by de correlating the input vectors. Then we have to calculate mutual information for all the independent components and then have to choose two components with the highest mutual information. The chosen components are subjected into fusion for much more detailed analysis and for optimal features. The fused image must be registered into the source image and the matching problem can be considered as a cost function based on the dissimilarity metric and that cost function must be low as possible and by optimizing the deformation field of the registration is obtained.

\section{IMAGE QUALITY METRICES}

The general requirements of an image fusing process are that it should preserve all valid and useful pattern information from the source images, while at the same time it should not introduce artifacts that could interfere with subsequent analyses. The performance measures used in this paper provide some quantitative comparison among different fusion schemes, mainly aiming at measuring the definition of an image.

\section{A. PEAK SIGNAL TO NOISE RATIO (PSNR)}

PSNR is the ratio between the maximum possible power of a signal and the power of corrupting noise that affects the fidelity of its representation [1] [2]. The PSNR measure is given by: 


\section{International Journal of Advanced Research in Electrical, Electronics and Instrumentation Engineering}

\section{(An ISO 3297: 2007 Certified Organization)}

\section{Vol. 3, Issue 9, September 2014}

$$
\operatorname{PSNR}(\mathrm{dB})=20 \log \frac{255 \sqrt{3 \mathrm{MN}}}{\sqrt{\sum_{\mathrm{i}=1}^{\mathrm{M}} \sum_{\mathrm{j}=1}^{\mathrm{N}}\left(\mathrm{B}^{\prime}(\mathrm{i}, \mathrm{j})-\mathrm{B}(\mathrm{i}, \mathrm{j})\right)^{2}}}
$$

Where, $B$ - the perfect image, $B^{\prime}$ - the fused image to be assessed, $i$ - pixel row index, $j$ - Pixel column index, M, NNo. of row and column

\section{B. ENTROPY (EN)}

Entropy is an index to evaluate the information quantity contained in an image. If the value of entropy becomes higher after fusing, it indicates that the information increases and the fusion performances are improved. Entropy is defined as:

$$
E=-\sum_{i=0}^{L-1} p_{i} \log _{2} p_{i}
$$

Where $\mathrm{L}$ is the total of grey levels, $p=\{p 0, p 1 \ldots p L-1\}$ is the probability distribution of each level [7].

\section{MEAN SQUARED ERROR (MSE)}

The mathematical equation of MSE is defined as:

$$
M S E=\frac{1}{m n} \sum_{i=1}^{m} \sum_{j=1}^{n}\left(A_{i j}-B_{i j}\right)^{2}
$$

Where, $A$ - the perfect image, $B$ - the fused image to be assessed, $i$ - pixel row index, $j$ - pixel column index, m, nNo. of row and columns.

\section{COMPARISON BETWEEN VARIOUS FUSION TECHNIQUES}

In the reference [2], we found that the value of the PSNR and Entropy in average method is less than as compared to value of other frequency domain method like SWT and laplacian method which means fused image are not exactly to registered image. That is why transform domain method are more suitable as compared to spatial domain method. But in some case spatial domain play a very important role in image fusion that contain high spatial information in fused image. In [1] a new fusion method based on combination of pixel and energy rule is proposed. On the basis of the study, only few comparisons between the different existing fusion techniques have been made and are analyzed theoretically which are shown in Table 1.

TABLE 1

\begin{tabular}{|l|l|l|l|l|}
\hline S.NO. & METHOD OF FUSION & DOMAIN & ADVANTAGES & DISADVANTAGES \\
\hline 1 & Simple Average & Spatial & Simplest method. & $\begin{array}{l}\text { The object is not clear } \\
\text { from a set of images. }\end{array}$ \\
\hline 2 & Simple Maximum & Spatial & Results in highly focused image. & $\begin{array}{l}\text { Blurring effect leads } \\
\text { to reduced contrast. }\end{array}$ \\
\hline 3 & PCA & Spatial & $\begin{array}{l}\text { Reduction of dimension results in } \\
\text { avoidance of unwanted data. }\end{array}$ & $\begin{array}{l}\text { Spatial distortions } \\
\text { exists. }\end{array}$ \\
\hline 4 & DWT & Transform & $\begin{array}{l}\text { Minimizes spatial distortions and also } \\
\text { PSNR is low. }\end{array}$ & $\begin{array}{l}\text { Spatial resolution is } \\
\text { less }\end{array}$ \\
\hline 5 & Cross Scale & Spatial & Color enhancement is done. & $\begin{array}{l}\text { Improper selection of } \\
\text { coefficients leads to } \\
\text { wrong diagnosis. }\end{array}$ \\
\hline 6 & Genetic Algorithm & Transform & Fused image is of High quality. & Reduced contrast \\
\hline
\end{tabular}




\title{
International Journal of Advanced Research in Electrical, Electronics and Instrumentation Engineering
}

\author{
(An ISO 3297: 2007 Certified Organization)
}

\section{Vol. 3, Issue 9, September 2014}

\section{CONCLUSION}

This paper performs the Comparative study of Image fusion techniques. On the basis of the study we have find out various issues in different techniques and to remove them, various techniques are proposed for fusion of different images. This review presents that which approach is better among all the existing Image Fusion techniques. Although selection of fusion algorithm is problem dependent but this review results that spatial domain provide high spatial resolution but spatial domain have image blurring problem. The fusion methods based on large decomposition levels are sensitive to noise, also it consumes more time as well as it requires more memory. And the spatial details cannot be captured well if the number of decomposition level is too small. The Wavelet transforms is the very good technique for the image fusion provide a high quality spectral content. But a good fused image have both quality so the combination of DWT \& spatial domain fusion method (like PCA) fusion algorithm improves the performance as compared to use of individual DWT and PCA algorithm. Finally this review concludes that an image fusion algorithm based on combination of Gabor wavelet transform and Shearlet transform may be the future trend of research regarding image fusion.

\section{REFERENCES}

1. M. N. Do and M. Vetterli, "Wavelet-based texture retrieval using generalized Gaussian density and Kullback-Leibler distance," IEEE Trans. Image Process, vol. 11, no. 2, pp. 146-158, Feb. 2002

M .Chandana,S. Amutha, and Naveen Kumar, “ A Hybrid Multi-focus Medical Image Fusion Based on Wavelet Transform”, International Journal of Research and Reviews in Computer Science (IJRRCS) Vol. 2, No. 4, August 2011.

S.L.Jany Shabu, Dr.C.Jayakumar, T.Surya "Survey of Image Fusion Techniques for Brain Tumor Detection”, IJEAT-Vol.3, No.2, December 2013.

V.P.S. Naidu and J.R. Raol "Pixel-level Image Fusion using Wavelets and Principal Component Analysis", Defence Science Journal, 2008. Smt.G. Mamatha (PhD), L.Gayatri, “An Image Fusion Using Wavelet and Curvelet Transform”, Global Journal of Advanced Engineering Technologies, Vol.1, Issue-2, 2012

10. M .Chandana,S. Amutha, and Naveen Kumar, - A Hybrid Multi-focus Medical Image Fusion Based on Wavelet Transform, International

11. Journal of Research and Reviews in Computer Science (IJRRCS), Vol. 2, No. 4, August 2011.

12. Shutao Li, Bin Yang, Jianwen Hu, "Performance comparison of different multi-resolution transforms for image fusion ", Elsevier-Information

13. Fusion, vol.12, no.2, 2011 